\title{
Nonlinear ion-acoustic structures in a nonextensive electron-positron-ion-dust plasma: Modulational instability and rogue waves ${ }^{\text {और }}$
}

\author{
Shimin Guo ${ }^{\mathrm{a}, \mathrm{c}}$, Liquan Mei ${ }^{\mathrm{a}, \mathrm{b}, *}$, Anbang Sun ${ }^{\mathrm{c}}$ \\ a School of Mathematics and Statistics, Xi'an Jiaotong University, Xi'an, 710049, China \\ ${ }^{\mathrm{b}}$ Center for Computational Geosciences, Xi'an Jiaotong University, Xi'an, 710049, China \\ ${ }^{\mathrm{c}}$ Research Group MAC, Centrum Wiskunde E Informatica, Amsterdam, 1098XG, The Netherlands
}

\section{A R T I C L E I N F O}

\section{Article history:}

Received 29 October 2012

Accepted 22 January 2013

Available online 4 February 2013

\section{Keywords:}

Electron-positron-ion-dust plasma

Nonlinear Schrödinger equation

Modulational instability

Ion-acoustic rogue wave

\begin{abstract}
A B S T R A C T
The nonlinear propagation of planar and nonplanar (cylindrical and spherical) ion-acoustic waves in an unmagnetized electron-positron-ion-dust plasma with two-electron temperature distributions is investigated in the context of the nonextensive statistics. Using the reductive perturbation method, a modified nonlinear Schrödinger equation is derived for the potential wave amplitude. The effects of plasma parameters on the modulational instability of ion-acoustic waves are discussed in detail for planar as well as for cylindrical and spherical geometries. In addition, for the planar case, we analyze how the plasma parameters influence the nonlinear structures of the first- and second-order ionacoustic rogue waves within the modulational instability region. The present results may be helpful in providing a good fit between the theoretical analysis and real applications in future spatial observations and laboratory plasma experiments.
\end{abstract}

(C) 2013 Elsevier Inc. All rights reserved.

\footnotetext{
The project is supported by NSF (10971164) and Doctoral Academic Freshman Award of Ministry of Education of China.

* Corresponding author at: School of Mathematics and Statistics, Xi'an Jiaotong University, Xi'an, 710049, China. Tel.: +86 29 82669051 .

E-mail addresses: gsm861@126.com (S. Guo), lqmei@mail.xjtu.edu.cn (L. Mei).
} 


\section{Introduction}

The electron-positron-ion plasma, characterized as a fully ionized gas containing electrons and positrons having equal masses and charges with opposite polarity, is believed to exist in many astrophysical objects such as active galactic nuclei [1], magnetospheres of pulsar [2], our early universe [3], and the inner regions of the accretion disks surrounding the black hole [4]. There is a possibility of the existence of astrophysical plasmas with a high concentration of positrons. From the view of large-scale structures in the cosmological environments, the positrons may have a high concentration and play an important role in the astrophysical plasmas. In the laboratory conditions, an electron-positron beam-plasma experiment has been performed with a large number of positrons [5] and the instability leading to a large amplitude has been also observed in this experiment. In 2008, physicists at the Lawrence Livermore National Laboratory in California produced more than 100 billion positrons [6], which could be valuable for the further laboratory plasma experiments with a long time existence and high concentration of positrons.

However, the dust grains ( $\mu \mathrm{m}$ to sub- $\mu \mathrm{m}$ sizes) are ubiquitous in the laboratory and space environments such as cometary surroundings, interstellar clouds and planetary rings $[7,8]$. When the dust grains are immersed into a plasma, they are usually charged due to the absorption of the charged particles. These charged dust grains could either modify the behavior of the normal waves and instabilities, or introduce new eigenmodes [9-11]. Therefore, the plasma may become an admixture of electrons, positrons, ions and dust grains. In the electron-positron-ion-dust (EPID) plasmas, the charge of the dust grains can be either negative or positive when the number of positrons depositing on them is either smaller or larger than the number of electrons. Accordingly, it is interesting and important to study ion-acoustic waves (IAWs) in the EPID plasmas [12,13].

It is well known that the properties of wave motions in plasma depend on the velocity distribution of the plasma particles. In the past few decades, the most commonly used distribution was the wellknown Maxwellian particle distribution. However, a number of space observations indicates the presence of particles which depart from the Maxwellian distribution. For example, the nonthermal and superthermal electrons have been observed in the Earth's bow-shock, in the upper ionosphere of Mars and in the vicinity of the Moon [14-17]. Also the non-Maxwellian electron velocity distributions have been observed and measured in the laboratory experiments [18]. Motivated by these findings, a nonextensive generalization of the Boltzmann-Gibbs-Shannon (BGS) entropy, first recognized by Renyi [19] and subsequently proposed by Tsallis [20], can suitably extend the standard additivity of the entropies to the nonextensive cases. In this context, the entropic index $q$ characterizes the degree of nonextensivity of the considered system. The q-nonextensive statistics can provide a powerful and convenient frame for the analysis of many astrophysical phenomena, such as the head-on collision of black holes, the dynamics of inflationary cosmologies and gravitational wave emission [21-23]. This distribution also presents a good fit to the experimental results. For electrostatic plane-wave propagation in a collisionless thermal plasma, the dispersion relation in Tsallis formalism fits the experimental data very well when $q<1$ [24], while the standard Bohm-Gross relation based on the classical Maxwellian distribution $(q=1)$ only provides a crude description. In Ref. [25], the anomalous diffusion and non-Gaussian statistics fitting the Tsallis distribution were detected experimentally in a two-dimensional driven-dissipative plasma system. It is necessary to point out that the transformation $\kappa=1 /(q-1)$ which was first provided by Leubner in Ref. [26] links $q$-statistic and $\kappa$-distribution [27] (note that for $q \rightarrow 1, \kappa \rightarrow \infty$ ).

Two-electron temperature plasmas are very common in the space [28], as well as in laboratory experiments [29]. For example, when out flows of the electron-positron plasma from pulsars enters an interstellar cold, low-density electron-ion plasma, the two-electron temperature plasma could be formed [30]. R. Sabry et al. studied the ion-acoustic envelope solitons in electron-positron-ion plasma with two-electron temperature distributions [31]. Also Mishra et al. investigated ion-acoustic double layers in electron-positron-ion plasma with two-electron temperature distributions [32].

Due to the carrier wave self-interaction or intrinsic medium nonlinearity, the propagation of nonlinear waves in a dispersive media is generally subject to amplitude modulation, which can be studied via the nonlinear Schrödinger equation (NLSE) derived by the reductive perturbation method (RPM) [33]. This method reduces the very complicated systems of equations modeling such complex 
behavior as wave propagation in the plasmas to one simple canonical forms and seeks to extract the effect of dominant nonlinearities.

The aim of this paper is to investigate the modulational instability (MI) of the planar and nonplanar IAWs in an EPID plasma with two-electron temperature distributions, whose components are either negatively or positively charged dust grains, positive ions, $q$-nonextensive positrons and electrons. For the planar case, the nonlinear evolution of the first- and second-order ion-acoustic rogue waves are presented in the paper. Rogue waves (also known as freak waves, killer waves, giant waves, or extreme waves), the singular, rare and high-energy phenomena with amplitude much higher than the average wave crests around it, have appeared in many physical systems such as oceans, Bose-Einstein condensates, optics, and super-fluids [34-37]. The rogue waves have been successively reported by numerous news media, Nature News, BBC News, Science Daily, Physics World, Scientific American, for instance. Due to the MI, the rogue waves may arise from the instabilities of the initial conditions that tend to grow exponentially and thus have the possibility of increasing up to very high amplitudes. It has been shown that the experimental results on the study of first- and second-order rogue waves [38-40] in a water tank which can be modeled by the NLSE are in good agreement with the theory. It is necessary to point out that the rational solutions of NLSE play an important role in the study of rogue waves. The first-order rational solution was given by Peregrine [41] as early as 1983. Based on [42], the next-order one was presented in Ref. [43] as a possible explanation for rogue waves with higher amplitude. In addition, this kind of rational solutions could resolve the mystery of rogue waves observed in optical fibers [44-46] and multicomponent plasma [47].

The paper is organized as follows: In Section 2, we present the basic equations of our theoretical model. Then, the modified NLSE is derived via the RPM. In Section 3, the effects of the plasma parameters on the MI and amplitudes of the first- and second-order ion-acoustic rogue waves are discussed. Finally, conclusions are given in Section 4.

\section{Basic equations and derivation of NLSE}

Let us consider a one-dimensional, unmagnetized plasma composed of positive ions, $q$-nonextensive positrons, negatively or positively fixed charged immobile dust grains, and two groups of $q$-nonextensive electrons having densities $n_{e c}$ and $n_{e h}$ with temperatures $T_{c}$ and $T_{h}$, respectively. Moreover, we assume that the phase velocity of the IAWs is much smaller than the electron and positron thermal velocities and larger than the ion thermal velocity, and positron annihilation time is larger than the inverse of the characteristic frequency of the IAWs. Overall charge neutrality at equilibrium is $n_{i 0}+n_{p 0}+s n_{d 0} Z_{d}=n_{e c 0}+n_{e h 0}$, where $n_{e c 0}, n_{e h 0}, n_{p 0}, n_{i 0}$, and $n_{d 0}$ are the equilibrium densities of two-electron components, positron component, ion component, and dust grain component, respectively. Either positive or negative dust charge can be accommodated in this description by setting $s=+1$ or -1 , respectively. The nonlinear behavior of IAWs in such EPID plasma can be in general described by the following set of normalized continuity, momentum, and Poisson's equations:

$$
\begin{aligned}
& \frac{\partial n_{i}}{\partial t}+\frac{1}{r^{\nu}} \frac{\partial}{\partial r}\left(r^{\nu} n_{i} u_{i}\right)=0, \\
& \frac{\partial u_{i}}{\partial t}+u_{i} \frac{\partial u_{i}}{\partial r}=-\frac{\partial \Phi}{\partial r}, \\
& \frac{1}{r^{\nu}} \frac{\partial}{\partial r}\left(r^{\nu} \frac{\partial \Phi}{\partial r}\right)=n_{e c}+n_{e h}-n_{p}-n_{i}-s \alpha,
\end{aligned}
$$

where $v=0,1,2$ for planar, cylindrical and spherical geometries, respectively. Here, $\alpha=Z_{d} n_{d 0} / n_{i 0}$, time and space coordinates $t$ and $r$ are in units of the ion plasma period $\omega_{p i}^{-1}=\sqrt{m_{i} /\left(4 \pi n_{i 0} e^{2}\right)}$ and Debye radius $\lambda_{D}=\sqrt{T_{\text {eff }} /\left(4 \pi n_{i 0} e^{2}\right)}$, and $e$ and $m_{i}$ are the magnitude of electronic charge and ion mass, respectively. The dependent variables $n_{i}$ and $u_{i}$ are the ion number density and fluid velocity normalized by equilibrium value $n_{i 0}$ and the effective ion acoustic velocity $C_{i}=\sqrt{T_{\text {eff }} / m_{i}}$, respectively. 


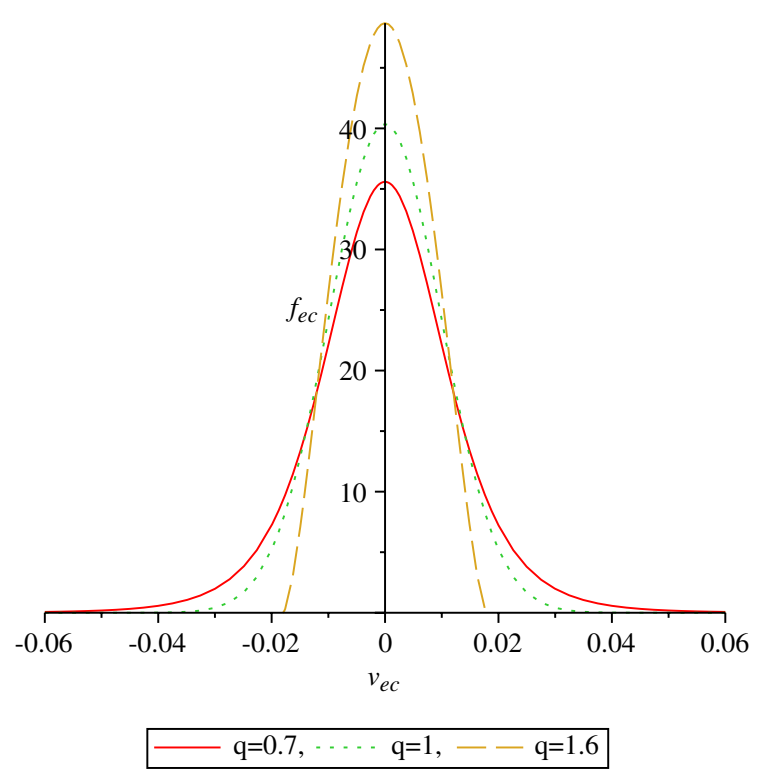

Fig. 1. Distribution function $f_{e c}\left(v_{e c}\right)$ against $v_{e c}$ for different values of $q$, with $T_{c}=0.00005 \mathrm{MeV}, \tilde{\Phi}=0$.

To model the effects of electron nonextensivity with temperature $T_{c}$, we refer to the following q-distribution function for one-dimensional case [48]:

$$
f_{e c}\left(v_{e c}\right)=C_{q}\left\{1-(q-1)\left[\frac{m_{e} v_{e c}^{2}}{2 T_{c}}-\frac{e \tilde{\Phi}}{T_{c}}\right]\right\}^{\frac{1}{q-1}}
$$

The constant $C_{q}$ is given by

$$
C_{q}= \begin{cases}n_{e c 0} \frac{\Gamma\left(\frac{1}{1-q}\right)}{\Gamma\left(\frac{1}{1-q}-\frac{1}{2}\right)} \sqrt{\frac{m_{e}(1-q)}{2 \pi T_{c}}}, & -1<q<1, \\ n_{e c 0}\left(\frac{1+q}{2}\right) \frac{\Gamma\left(\frac{1}{q-1}+\frac{1}{2}\right)}{\Gamma\left(\frac{1}{q-1}\right)} \sqrt{\frac{m_{e}(q-1)}{2 \pi T_{c}}}, & q>1 .\end{cases}
$$

Here, $v_{e c}, \Gamma(\cdot), \tilde{\Phi}$, and $m_{e}$ denote the speed of electrons, Gamma function, the electrostatic potential, and the mass of electron, respectively. It is easy to see that: (i) when $q \rightarrow 1$, the above particle velocity distribution function reduces to the Maxwell-Boltzmann distribution function. (ii) when $q<-1$, the above distribution is unnormalizable. For $q>1$, the above distribution function exhibits a thermal cutoff on the maximum value allowed for the velocity of the particles, which is given by

$$
v_{\max }=\sqrt{\frac{2 T_{c}}{m_{e}(q-1)}-\frac{2 e \tilde{\Phi}}{m_{e}}} .
$$

For a better understanding of the physical meaning of the parameter $q$, we illustrate the distribution function $f_{e c}\left(v_{e c}\right)$ for different values of $q$ in Fig. 1. Form this figure, we can see that: (i) the height and shape of the velocity distribution change remarkably for different $q$ values. (ii) there is a velocity cutoff beyond which no possible states exist when $q>1$. 
Integrating the q-distribution function $f_{e c}\left(v_{e c}\right)$ over all the velocity space and adopting the normalization, we have the following normalized density

$$
n_{e c}=\mu\left[1+(q-1) \frac{1}{\mu+\eta \beta} \Phi\right]^{\frac{q+1}{2(q-1)}} .
$$

Similarly, we have the following normalized electron density with temperature $T_{h}$

$$
n_{e h}=\eta\left[1+(q-1) \frac{\beta}{\mu+\eta \beta} \Phi\right]^{\frac{q+1}{2(q-1)}},
$$

for the positron we obtain the following normalized density

$$
n_{p}=\delta[1-(q-1) \gamma \Phi]^{\frac{q+1}{2(q-1)}} .
$$

In the above equations, $\mu=n_{e c 0} / n_{i 0}, \eta=n_{e h 0} / n_{i 0}, \delta=n_{p 0} / n_{i 0}, \beta=T_{c} / T_{h}, \gamma=T_{\text {eff }} / T_{p}, T_{\text {eff }}=$ $T_{c} /(\mu+\eta \beta), \Phi=e \widetilde{\Phi} / T_{\text {eff }}, T_{p}$ is the positron temperature. The charge-neutrality condition is expressed as

$$
\mu+\eta=\delta+s \alpha+1 .
$$

To investigate the modulation of IAWs propagating in our nonextensive plasma, we apply the RPM to Eqs. (1)-(3) and (7)-(9). The independent variables are stretched as

$$
\xi=\epsilon(r-V t), \quad \tau=\epsilon^{2} t
$$

where $\epsilon$ is a real small dimensionless expansion parameter and $V$ is the wave group velocity to be determined later.

The dependent variables are expanded as

$$
\begin{aligned}
& n_{i}=1+\sum_{k=1}^{\infty} \epsilon^{k} \sum_{l=-\infty}^{+\infty} n_{l}^{(k)}(\xi, \tau) e^{i l(k r-\omega t)}, \\
& u_{i}=\sum_{k=1}^{\infty} \epsilon^{k} \sum_{l=-\infty}^{+\infty} u_{l}^{(k)}(\xi, \tau) e^{i l(k r-\omega t)} \\
& \Phi=\sum_{k=1}^{\infty} \epsilon^{k} \sum_{l=-\infty}^{+\infty} \Phi_{l}^{(k)}(\xi, \tau) e^{i l(k r-\omega t)}
\end{aligned}
$$

Here, $i=\sqrt{-1}, k$ and $\omega$ are real variables representing the fundamental wave number and frequency, respectively. Since $n_{i}, u_{i}$ and $\Phi$ must be real, the coefficients in Eq. (12) satisfy the condition $A_{-l}^{(k)}=$ $A_{l}^{(k) *}$, and the asterisk denotes complex conjugate.

Substituting Eqs. (11) and (12) into Eqs. (1)-(3) and (7)-(9), and collecting the terms in different powers of $\epsilon$, the first order $(k=1)$ equations with $l=1$, give

$$
n_{1}^{(1)}=\frac{k^{2}}{\omega^{2}} \Phi_{1}^{(1)}, \quad u_{1}^{(1)}=\frac{k}{\omega} \Phi_{1}^{(1)} .
$$

Thus we obtain the following dispersion relation

$$
\frac{\omega^{2}}{k^{2}}=\frac{1}{\left(c_{1} \delta \gamma+c_{1} U \mu+c_{1} W \eta+k^{2}\right)},
$$

where $U=\frac{1}{\mu+\eta \beta}, W=\frac{\beta}{\mu+\eta \beta}, c_{1}=\frac{(q+1)}{2}$. 
Using the first-order quantities, the second order $(k=2)$ equations with $l=1$ are

$$
\begin{aligned}
& n_{1}^{(2)}=\frac{i k}{\omega^{3}}\left[(-2 \omega+2 k V) \frac{\partial \Phi_{1}^{(1)}}{\partial \xi}-i k \omega \Phi_{1}^{(2)}\right], \\
& u_{1}^{(2)}=\frac{i}{\omega^{2}}\left[(-\omega+k V) \frac{\partial \Phi_{1}^{(1)}}{\partial \xi}-i k \omega \Phi_{1}^{(2)}\right],
\end{aligned}
$$

with the compatibility condition

$$
V=\frac{\partial \omega}{\partial k}=(\delta \gamma+U \mu+W \eta) c_{1} \frac{\omega^{3}}{k^{3}} .
$$

We recall that the above condition is the group velocity of the waves.

The second harmonic modes $(k=l=2)$ which arise from the nonlinear self-interaction of the carrier waves, are obtained in terms of $\left(\Phi_{1}^{(1)}\right)^{2}$ as

$$
\begin{aligned}
& n_{2}^{(2)}=\Delta_{1}\left(\Phi_{1}^{(1)}\right)^{2}, \\
& u_{2}^{(2)}=\Delta_{2}\left(\Phi_{1}^{(1)}\right)^{2}, \\
& \Phi_{2}^{(2)}=\Delta_{3}\left(\Phi_{1}^{(1)}\right)^{2},
\end{aligned}
$$

where $\Delta_{1}, \Delta_{2}$ and $\Delta_{3}$ are given in the Appendix.

The nonlinear self-interaction of the carrier waves also result in the creation of a zeroth harmonic. Its strength is analytically determined by taking into account the $l=0$ component of the third-order expanded equations which can be expressed as

$$
\begin{aligned}
& n_{0}^{(2)}=\Delta_{4}\left|\Phi_{1}^{(1)}\right|^{2}, \\
& u_{0}^{(2)}=\Delta_{5}\left|\Phi_{1}^{(1)}\right|^{2}, \\
& \Phi_{0}^{(2)}=\Delta_{6}\left|\Phi_{1}^{(1)}\right|^{2},
\end{aligned}
$$

where $\Delta_{4}, \Delta_{5}$ and $\Delta_{6}$ are given in the Appendix.

Finally, with the aid the above derived equations (13)-(19), the third harmonic modes $(k=3$ and $l=1$ ) give a system of equations which can be reduced to the following modified NLSE:

$$
i \frac{\partial \Phi}{\partial \tau}+P \frac{\partial^{2} \Phi}{\partial \xi^{2}}+Q|\Phi|^{2} \Phi+i \frac{v}{2 \tau} \Phi=0
$$

Here, $\Phi_{1}^{(1)}$ is written as $\Phi$ for simplicity. The term $i \frac{v}{2 \tau} \Phi$ appearing in Eq. (20) is due to the nonplanar (cylindrical and spherical) geometry effects. The dispersion coefficient $P$ and nonlinear coefficient $Q$ are

$$
\begin{aligned}
P= & \frac{1}{2} \frac{d \omega^{2}}{d k^{2}}=\frac{-4 V \omega k-\omega^{4}+\omega^{2}+3 k^{2} V^{2}}{2 k^{2} \omega} \\
Q= & \frac{1}{2 k^{2}}\left[-k^{2} \omega\left(\Delta_{1}+\Delta_{4}\right)-2 k^{3}\left(\Delta_{2}+\Delta_{5}\right)+c_{2}\left(\Delta_{3}+\Delta_{6}\right)\left(2 \omega^{3} \eta W^{2}-2 \omega^{3} \delta \gamma^{2}\right.\right. \\
& \left.\left.+2 \omega^{3} \mu U^{2}\right)+c_{3}\left(3 \omega^{3} \delta \gamma^{3}+3 \omega^{3} \eta W^{3}+3 \omega^{3} \mu U^{3}\right)\right],
\end{aligned}
$$

where $U=\frac{1}{\mu+\eta \beta}, W=\frac{\beta}{\mu+\eta \beta}, c_{2}=\frac{(q+1)(3-q)}{8}, c_{3}=\frac{(q+1)(q-3)(3 q-5)}{48}, \Delta_{1}-\Delta_{6}$ are given in the Appendix. 


\section{Results and discussions}

\subsection{Nonlinear dispersion relation}

To study the MI of IAWs in our plasma, we consider the development of small modulation $\delta \varphi=$ $\delta \varphi(\tau, \xi)$ according to

$$
\Phi=\left(\Phi_{0}+\delta \varphi\right) \exp \left[-i \int_{\tau_{0}}^{\tau} \Delta(\tau) d \tau-\frac{v}{2} \ln \tau\right],
$$

where $\Phi_{0}$ is the constant (real) amplitude of pump carrier wave which is much larger than the perturbation, i.e., $\Phi_{0} \gg|\delta \varphi|, \Delta(\tau)$ is a nonlinear frequency shift produced by nonlinear reaction.

Substituting Eq. (22) into Eq. (20) and collecting the terms in the zeroth and first order, we have

$$
\begin{aligned}
& \Delta(\tau)=-\frac{Q\left|\Phi_{0}\right|^{2}}{\tau^{\nu}}, \\
& i \frac{\partial \delta \varphi}{\partial \tau}+P \frac{\partial^{2} \delta \varphi}{\partial \xi^{2}}+\frac{Q\left|\Phi_{0}\right|^{2}}{\tau^{\nu}}\left(\delta \varphi+\delta \varphi^{*}\right)=0,
\end{aligned}
$$

where $\delta \varphi^{*}$ is the complex conjugate of $\delta \varphi$. Then, substituting $\delta \varphi(\tau, \xi)=U(\tau, \xi)+i V(\tau, \xi), U=$ $\operatorname{Re}\left[U_{0} e^{i\left(K \xi-\int_{\tau_{0}}^{\tau} \Omega(\tau) d \tau\right)}\right], V=\operatorname{Re}\left[V_{0} e^{i\left(K \xi-\int_{\tau_{0}}^{\tau} \Omega(\tau) d \tau\right)}\right]$ into Eq. (24), we can get the following nonlinear dispersion relation $[31,49]$ for the amplitude modulation of the IAWs modes:

$$
\Omega^{2}=\left(P K^{2}\right)^{2}\left(1-\frac{Q}{P} \frac{2\left|\Phi_{0}\right|^{2}}{\tau^{v}} \frac{1}{K^{2}}\right) .
$$

Here, $\left(K \xi-\int_{\tau_{0}}^{\tau} \Omega(\tau) d \tau\right)$ is the modulation phase with $K(\ll k)$ and $\Omega(\ll \omega)$ are the perturbation wave number and frequency of the modulation, respectively. One can immediately see that the MI condition will be satisfied if $P Q>0$ and $K^{2} \leq K_{c}^{2}=\frac{2\left|\Phi_{0}\right|^{2} Q}{P \tau^{v}}$. It would be interesting to investigate the dependence of MI growth rate on the nonextensive index $q$. From Eq. (25), we define the MI growth rate $\Gamma$ as

$$
\Gamma=a X\left(2 \frac{b}{a \tau^{\nu}}-X^{2}\right)^{\frac{1}{2}},
$$

where $a=\left|\frac{P}{P_{1}}\right|, b=\left|\frac{Q}{Q_{1}}\right|, X=\frac{K}{\left|\Phi_{0}\right|} \sqrt{\frac{P_{1}}{Q_{1}}}$. Here, $P_{1}$ and $Q_{1}$ are the coefficients $P$ and $Q$ expressed by Eq. (21) in the Maxwellian case $(q \rightarrow 1)$. Obviously, the growth rate $\Gamma$ is significantly affected by the variation in values of $q$.

From Eqs. (21) and (10), it is easy to see that the coefficients $P$ and $Q$ are the functions of the plasma parameters $k, q, \mu, \gamma, \delta, \beta$ and $\alpha$, which means that these parameters could change the sign of product $P Q$. Since the effects of parameters $\beta, \delta$ and $\gamma$ on critical wave number threshold $k_{c}$ have been discussed in a electron-positron-ion plasma in Ref. [31], we just investigate the effects of the nonextensive index $q$, dust concentration $\alpha$, wave number $k$ and cold electron to positive ion density ratio $\mu$ on the MI and rogue waves in this paper.

\subsection{MI of planar excitations and rogue waves}

When setting $v=0$ in Eqs. (20), (25) and (26), we can get the NLSE, nonlinear dispersion relation and MI growth rate for the planar envelope excitations, respectively.

It is easy to see that IAWs are stable for $P Q<0$ in the presence of a small perturbation and the IAWs may propagate in the form of dark-type excitations [50], manifested as localized envelope holes or voids in the center, amidst a uniform wave energy region. In other words, these excitations may either reach a vanishing or finite amplitude in the center, corresponding to the dark or gray 
solitons, respectively. On the other hand, when $P$ and $Q$ have the same sign ( $P Q>0)$, the carrier wave is modulationally unstable, which may collapse or blow up due to the external perturbations, or lead to the bright-type envelope modulated wave packets [50], i.e., the localized pulse-shaped envelopes confining the fast carrier wave. For this unstable case, small amplitude waves may grow to higher amplitudes and then the rogue waves may be generated in a relatively small region. It is easy to see that the MI growth rate of the planar IAWs presents the maximum value $\Gamma_{\max }=b$, i.e. $\operatorname{Im} \Omega=|Q|\left|\Phi_{0}\right|^{2}$, at the point $X=\sqrt{\frac{b}{a}}$. Then, the instability window becomes the interval $\left[0, \sqrt{\frac{2 b}{a}}\right]$.

Based on the above findings, we can see that it is important to analyze how the plasma parameters influence the sign of $P Q$ which indicates where the stable $(P Q<0)$ and unstable $(P Q>0)$ regions set in. Fig. 2 depicts the effects of the plasma parameters $q, \alpha$ and $\mu$ on the critical wave number threshold $k_{c}$ for which $P Q$ changes its sign in the $(q, k),(\alpha, k)$, and $(\mu, k)$-planes as follows:

(i) from plot (a), we can see that for $s=-1$ and $-1<q<0.35$, increasing the values of $q$ leads to a decrease of the critical wave number $k_{c}$ until $q$ reaches a certain value $q=q_{c} \approx 0.35$, i.e. increase the $q$ value could change $P Q<0$ (stable case) into $P Q>0$ (unstable case) when $q<q_{c}$. Then, further increasing the values of $q$ beyond $q_{c}$ could increase the values of $k_{c}$, i.e. increase the values of $q>q_{c}$ could change $P Q>0$ (unstable case) into $P Q<0$ (stable case). For $s=+1$, increasing $q$ value has the similar qualitative effect on $k_{c}$, which is shown in plot (b). Comparing the plots (a) and (b), it can be seen that the $k_{c}$ value at $q_{c}$ for the case $s=-1$ is smaller than that for the case $s=+1$.

(ii) Plots (c) and (d) show the effects of parameter $\alpha$ on the stable/unstable regions of the EPID plasma containing negative and positive dust, respectively. It is noticed that increasing the negative dust concentration $\alpha$ leads to a decrease of the critical wave number $k_{c}$, i.e. an increase in $\alpha$ could change $P Q<0$ (stable case) into $P Q>0$ (unstable case). However, the effect of positive dust concentration $\alpha$ is opposite to that of the negative case. In other words, an increase in positive dust concentration $\alpha$ increases the $k_{c}$ value and change $P Q>0$ (unstable case) into $P Q<0$ (stable case), which means that the modulationally unstable region of the system containing negative dust is bigger than that of the system containing positive dust.

(iii) Plot (e) shows that, for the case $s=-1$ increasing the values of $\mu$ leads to a decrease of the critical wave number $k_{c}$ until $\mu$ reaches a certain value $\mu=\mu_{c} \approx 0.2$, and further increasing the values of $\mu$ beyond $\mu_{c}$ could increase the values of $k_{c}$. In other words, an increase in $\mu<\mu_{c}$ could change $P Q<0$ (stable case) into $P Q>0$ (unstable case), while the effect of $\mu>\mu_{c}$ on the stable/unstable region is opposite to that of $\mu<\mu_{c}$. For the case $s=+1$, plot (f) shows that the parameter $\mu$ has the similar qualitative effect on the stable/unstable regions. From these two plots, we can see that the unstable (stable) region for $s=-1$ is bigger (smaller) than that for $s=+1$.

In Fig. 3, we present the effect of nonextensive parameter $q$ on the MI growth rate $\Gamma$ of the EPID plasma containing negative dust $(s=-1)$. Obviously, the MI growth rate is significantly affected by the variation in the values of $q$. From this figure, we can draw the conclusion that for a fixed value of $X$, increasing the values of $q$ in the interval $-1<q<q_{c}$ could lead to an increase in the growth rate $\Gamma$; then, beyond a certain value $q=q_{c}$, the influence of $q$ on the growth rate is opposite to that of $q \in\left(-1, q_{c}\right)$, i.e., the growth rate decreases as the values of $q$ increase in the interval $q>q_{c}$. For $s=+1$, the parameter $q$ has the same qualitative behavior on the MI growth rate $\Gamma$ as Fig. 3 . For simplicity, we do not present the figure here.

Now, we will analyze how the plasma parameters $k, \mu, \alpha$ and $q$ affect the amplitude of the following first-order ion-acoustic rogue wave (Peregrine breather) [41]

$$
\Phi(\xi, \tau)=\sqrt{\frac{2 P}{Q}}\left(\frac{4+16 i P \tau}{1+16(P \tau)^{2}+4 \xi^{2}}-1\right) e^{(2 i P \tau)},
$$

which can be understood as a limiting case of Kuznetsov-Ma breather [51,52] and Akhmediev breather [53]. Fig. 4 shows that, the rational solution (27) breathes only once and describes a single 
a

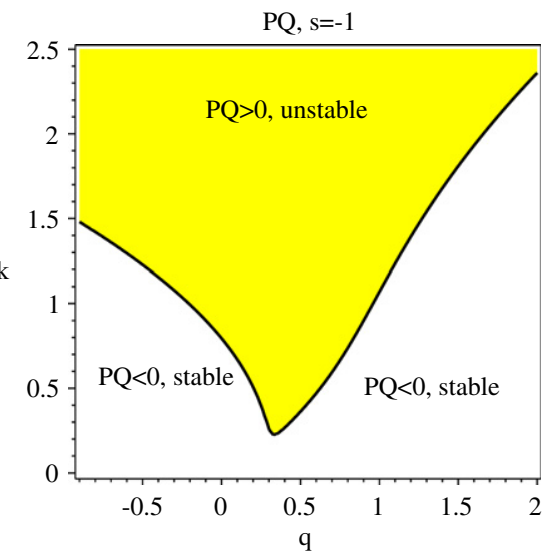

$\mathrm{C}$

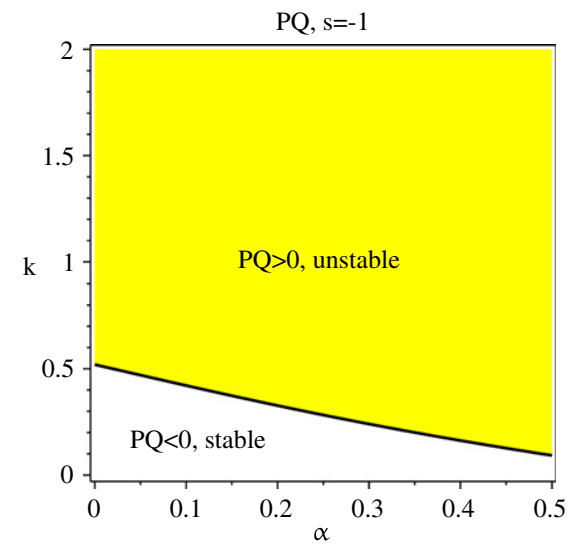

e

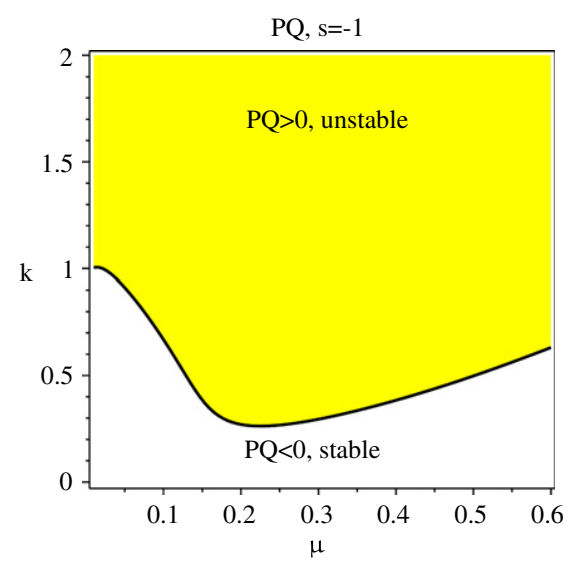

b

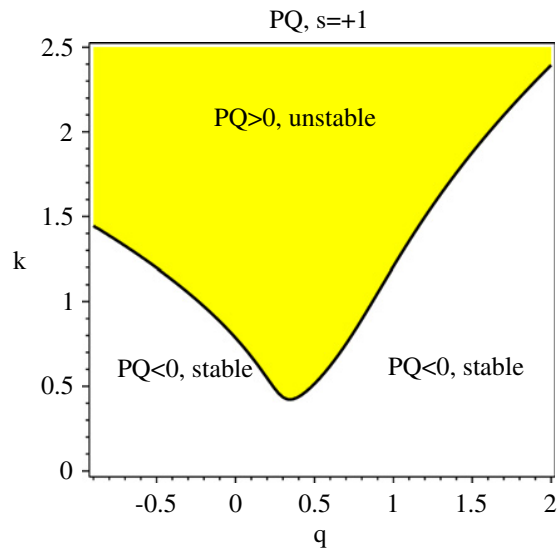

d

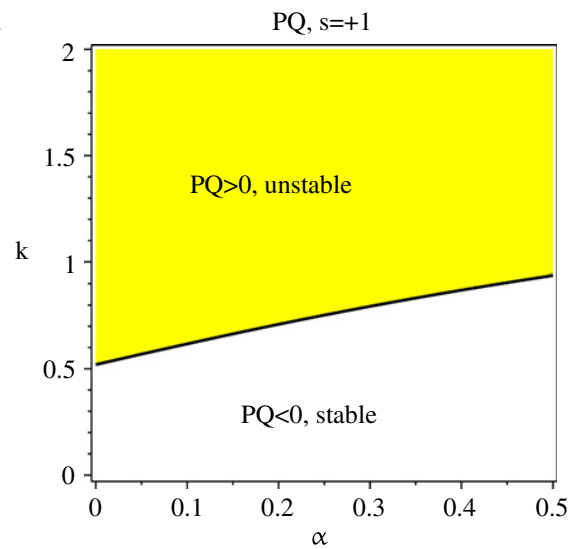

f

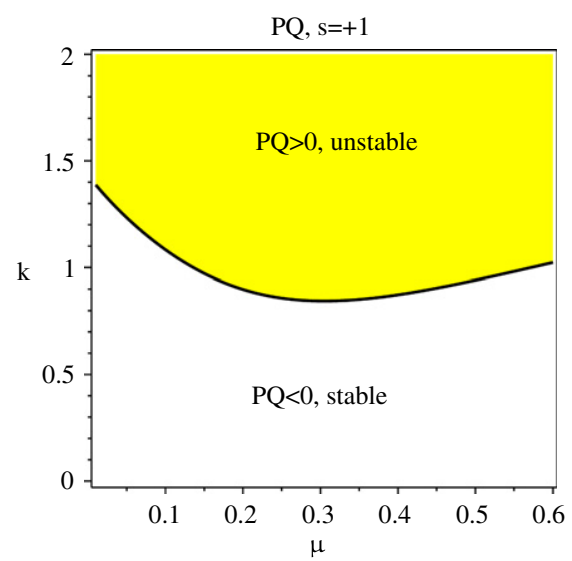

Fig. 2. The contour of product $P Q$. The yellow color represents the modulationally unstable region $(P Q>0)$, and white color represents the stable region $(P Q<0)$. The parameters are: $(q, k)$-plane for $\alpha=0.2, \beta=0.06, \gamma=0.5, \mu=0.6, \delta=0.1$, (a) $s=-1$, and (b) $s=+1$; $(\alpha, k)$-plane for $\beta=0.2, \gamma=0.05, \mu=0.55, \delta=0.05, q=0.6$, (c) $s=-1$, and (d) $s=+1$; ( $\mu, k$ )-plane for $\alpha=0.2, \beta=0.2, \gamma=0.01, \delta=0.05, q=0.8$, (e) $s=-1$, and (f) $s=+1$. (For interpretation of the references to colour in this figure legend, the reader is referred to the web version of this article.) 


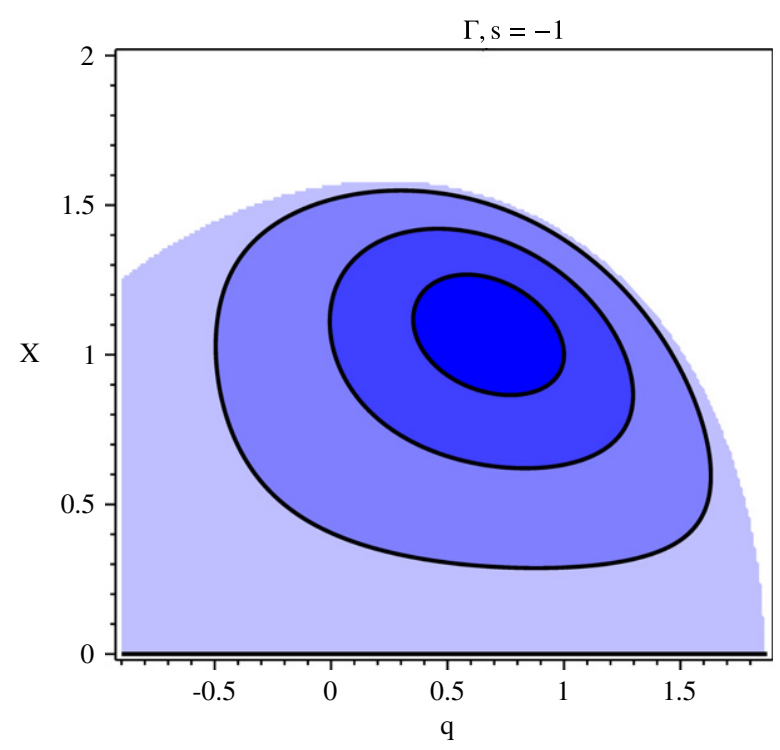

Fig. 3. The contour of MI growth rate $\Gamma$. The lighter-color regions correspond to the higher values of $\Gamma$. The parameters are: $\alpha=0.4, \beta=0.2, \gamma=0.03, \mu=0.5, \delta=0.05, k=2, s=-1$.

a

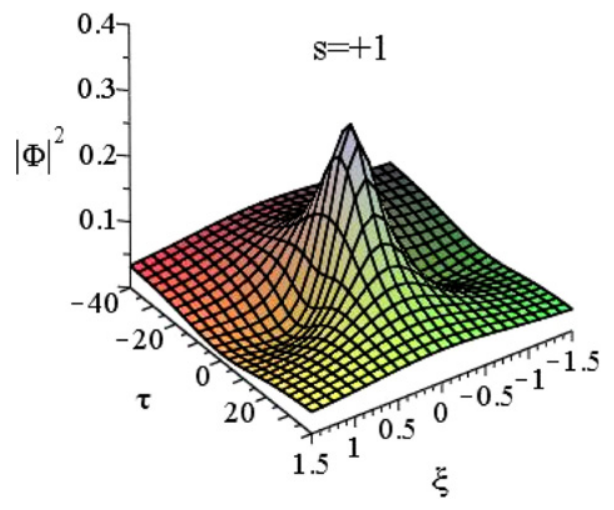

b

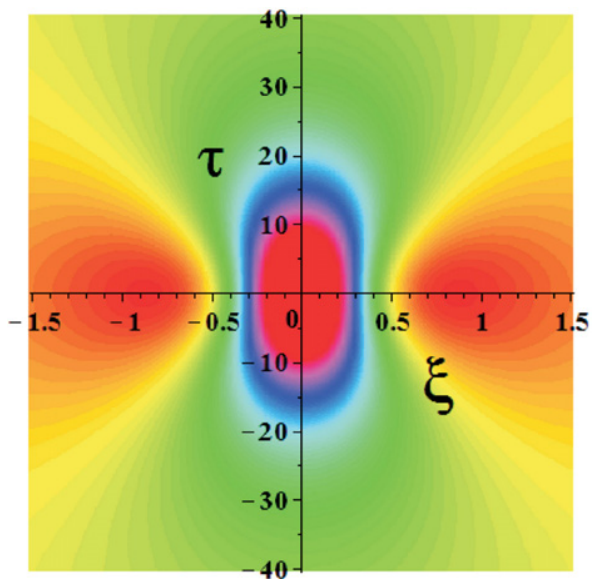

Fig. 4. Wave propagation (a) and density plot (b) for the electrostatic potential $|\Phi|^{2}$ of the first-order ion-acoustic rogue wave solution (27). The parameters are: $\alpha=0.2, \beta=0.02, \gamma=0.3, \mu=0.6, \delta=0.1, q=1.6, k=2.8$, and $s=+1$.

wave that appears from nowhere and then disappears without a trace. In other words, this ionacoustic rogue wave solution is able to concentrate a significant amount of the IAWs energy in a relatively small region. This property is typical for the rogue waves.

The effects of the dust concentration $\alpha$ and nonextensive distribution parameter $q$ on the rational solution (27) are depicted in Fig. 5 for both the cases $s=-1$ and $s=+1$. Clearly, increasing the values of $q$ leads to enhance the amplitude of the first-order ion-acoustic rogue waves for these two cases. Plot (a) shows that an increase in negative dust concentration $\alpha$ could slightly shrink the nonlinearity of the considered system and make the pulses shorter, while plot (b) shows the pulses become slightly taller for the higher values of positive dust concentration $\alpha$. 
a

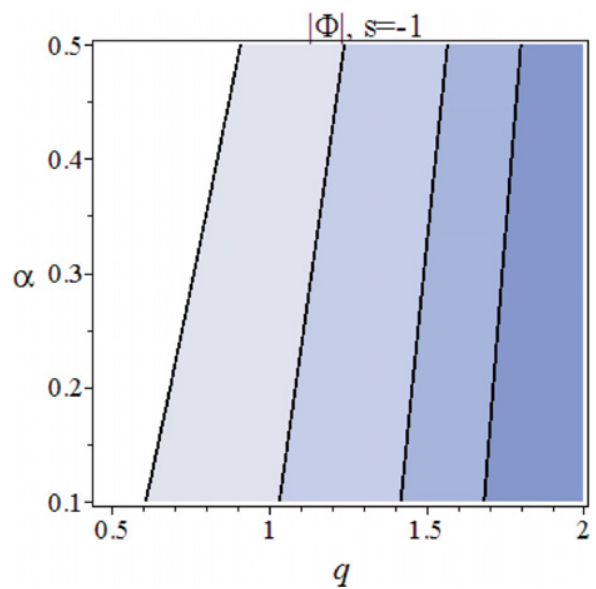

$\mathrm{b}$

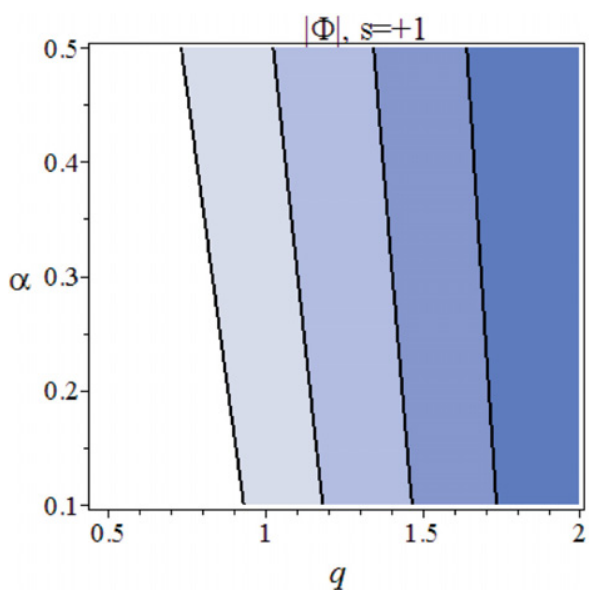

Fig. 5. The contour of the first-order ion-acoustic rogue wave amplitude. The lighter-color regions correspond to the higher values of amplitude. The parameters are: $\beta=0.1, \gamma=0.01, \mu=0.5, \delta=0.01, k=2.5$, (a), $s=-1$, (b), $s=+1$.

a

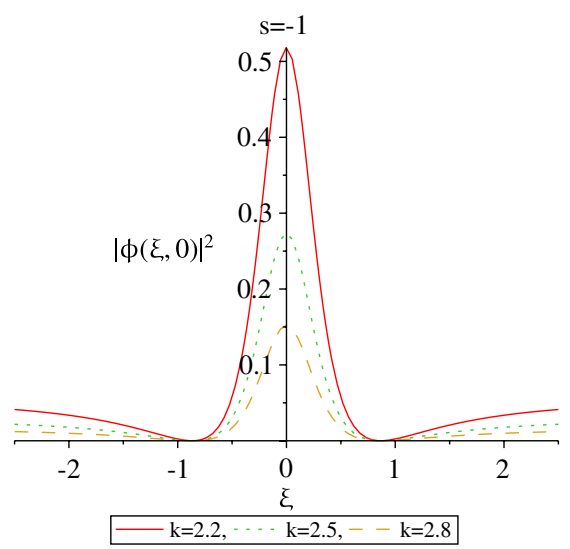

$\mathrm{b}$

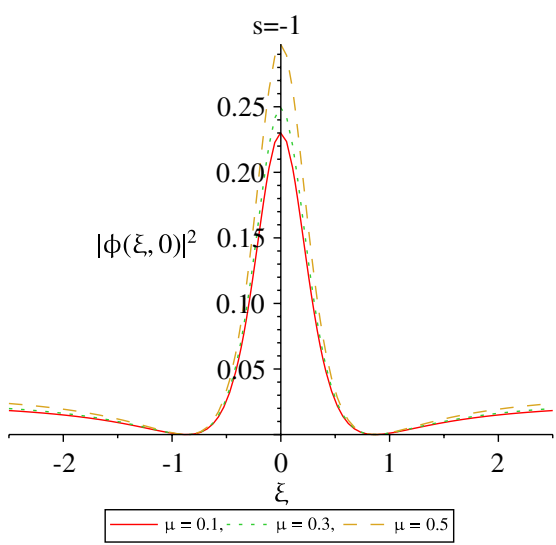

Fig. 6. The absolute values of the electrostatic potential $\Phi$ represented by Eq. (27) for different values of wave number $k$ (a) and cold electron to positive ion density ratio $\mu$ (b). The parameters are: $\alpha=0.3, \beta=0.1, \gamma=0.05, \delta=0.05, q=0.7, s=-1$, (a) $\mu=0.4$, (b) $k=2.5$.

To see how the parameters $k$ (wave number) and $\mu$ (cold electron to positive ion density ratio) affect the first-order ion-acoustic rogue waves, we present the variation of the absolute values $\Phi(\xi, 0)$ for different values of these two parameters in Fig. 6. Plot (a) shows that, for the case $s=-1$ the amplitudes of the ion-acoustic rogue waves decrease when the values of $k$ increase. However, the effect of $\mu$ on the amplitudes of the ion-acoustic rogue waves is opposite to that of $k$, i.e. increasing the values of $\mu$ could increase the rogue wave amplitude. This interesting phenomena may be explained as follows: an increase in $k(\mu)$ could shrink (enhance) the nonlinearity of the plasma system and disperse (concentrate) its energy, which makes the pulses shorter (taller). For the case $s=+1$, the parameters $k$ and $\mu$ have the same qualitative behavior on the ion-acoustic rogue waves as plots (a) and (b), respectively. For simplicity, we do not include the figure here.

Since the actual wave dynamic consists of a nonlinear superposition of many simple solutions and two or more Peregrine solutions can be combined into a more complicated doubly-localized structures with a higher amplitude, it is interesting and important to investigate the following second-order ion- 
a

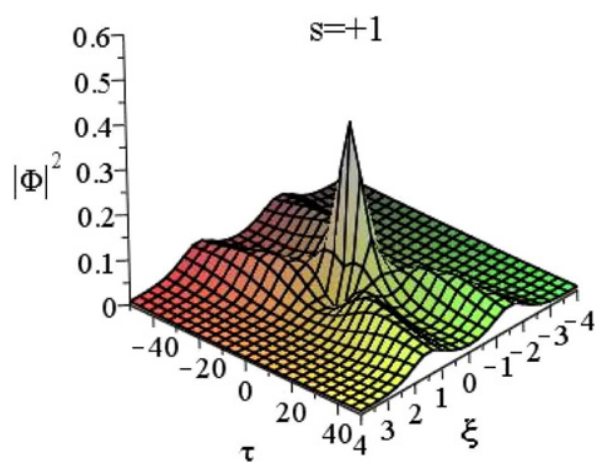

b

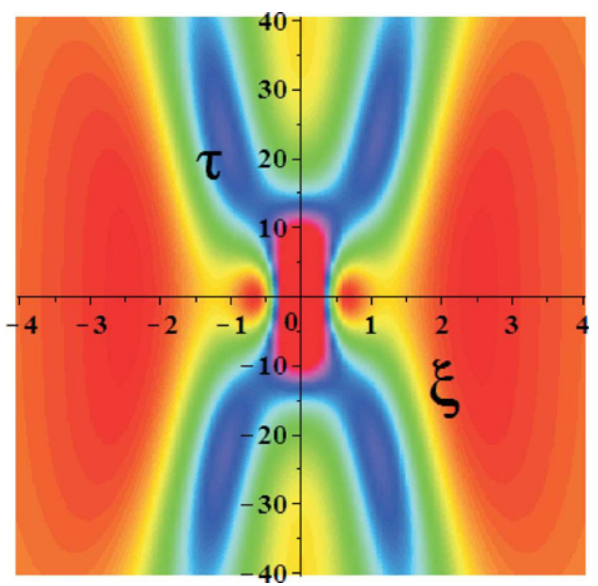

Fig. 7. Wave propagation (a) and density plot (b) for the electrostatic potential $|\Phi|^{2}$ of the second-order ion-acoustic rogue wave solution (28). The parameters are the same as that in Fig. 4.

acoustic rogue wave [54]:

$$
\Phi(\xi, \tau)=\sqrt{\frac{P}{Q}}\left(1+\frac{G_{2}(\xi, \tau)+i K_{2}(\xi, \tau)}{D_{2}(\xi, \tau)}\right) e^{(i P \tau)},
$$

where

$$
\begin{aligned}
G_{2}(\xi, \tau)= & -\frac{1}{2} \xi^{4}-6(P \xi \tau)^{2}-10(P \tau)^{4}-\frac{3}{2} \xi^{2}-9(P \tau)^{2}+\frac{3}{8} \\
K_{2}(\xi, \tau)= & -P \tau\left[\xi^{4}+4(P \xi \tau)^{2}+4(P \tau)^{4}-3 \xi^{2}+2(P \tau)^{2}-\frac{15}{4}\right] \\
D_{2}(\xi, \tau)= & \frac{1}{12} \xi^{6}+\frac{1}{2} \xi^{4}(P \tau)^{2}+\xi^{2}(P \tau)^{4}+\frac{2}{3}(P \tau)^{6}+\frac{1}{8} \xi^{4}+\frac{9}{2}(P \tau)^{4} \\
& -\frac{3}{2}(P \xi \tau)^{2}+\frac{9}{16} \xi^{2}+\frac{33}{8}(P \tau)^{2}+\frac{3}{32} .
\end{aligned}
$$

For a better understanding, we present the above second-order ion-acoustic rogue wave in Fig. 7. It is easy to see that this rational solution is also localized both in $\tau$ and $\xi$ directions and has a higher amplitude than the first-order case.

Fig. 8 depicts that, the amplitude of the rogue wave is pronounced affected by the nonextensive distribution parameter $q$ and wave number $k$ for $s=-1$. It is revealed that, for a fixed value of $k$, increasing $q$ could decrease the amplitude when $-1<q<q_{c}$. Then, beyond the critical value $q_{c}$, a further increase in $q$ leads to an increase in the amplitude. We speculate this interesting phenomenon could be explained as follows: increasing the values of nonextensive parameters $q \in\left(-1, q_{c}\right)$ could not enhance the nonlinearity of the system and shrink its energy, then the pulses become shorter; for $q>q_{c}$, an increase in $q$ would lead to an enhancement of the nonlinearity of the plasma and concentrate a significant amount of energy, which makes the pulses taller. Furthermore, the amplitude of the ion-acoustic rogue wave decreases with an increase in wave number $k$, which means that increasing the $k$ value reduces the nonlinearity and disperses the energy of the system. For $s=+1$, the parameters $q$ and $k$ have same qualitative influence on the amplitude as Fig. 8 , and therefore we do not include the figure here.

Fig. 9 shows the effects of parameters $\mu$ and $\alpha$ on the amplitude of ion-acoustic rogue wave for both the cases $s=-1$ and $s=+1$. Here, we consider two possible ranges of the nonextensive parameter $q$, viz., $-1<q<0$ and $q>0$. It can be seen from this figure that: 


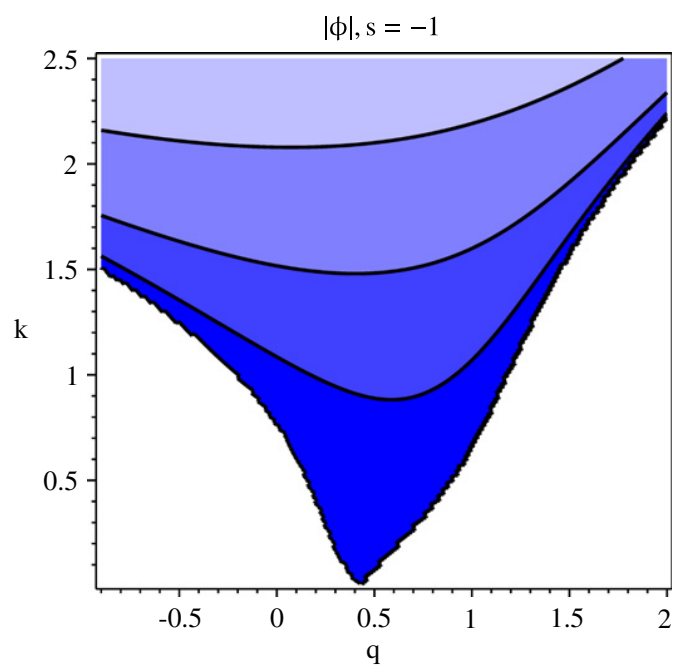

Fig. 8. The contour of the second-order ion-acoustic rogue wave amplitude. The lighter-color regions correspond to the higher values of amplitude. The parameters are: $\alpha=0.4, \beta=0.1, \gamma=0.01, \mu=0.6, \delta=0.01$, and $s=-1$.

(i) for $s=-1$ and $-1<q<0$, increasing $\mu$ would lead to shrinking the rogue wave amplitude, which is presented in plot (a). In other words, increasing $\mu$ reduces the nonlinearity of the system and disperses its energy, so the pulses become shorter. Plot (b) shows that, increasing $\mu$ could decrease the amplitude when $\mu<\mu_{c}$ and $q>0$; then, beyond the critical value $\mu_{c}$, a further increase in $\mu$ leads to an increase of the amplitude. This behavior may be explained as follows: increasing the values of $\mu<\mu_{c}$ would not enhance the nonlinearity of the plasma, then the pulses become shorter; for $\mu>\mu_{c}$, an increase in $\mu$ could lead to an enhancement of the nonlinearity of the system and concentrate a significant amount of energy, which makes the pulses become taller.

(ii) From plots (c) and (d), it is clear to see that for $s=+1$, i.e., the plasma containing positive dust, parameter $\mu$ has the same effect on the amplitude as the case $s=-1$.

(iii) For $s=-1$ and $-1<q<0$, increasing $\alpha$ could lead to an increase of the amplitude if the value of $\mu$ is less than a certain value $\mu_{c}$ (Note that this value is different from the one in the above discussion (i).); however, when $\mu>\mu_{c}$ increasing $\alpha$ could lead to a decrease of the amplitude. This interesting behavior is shown in plot (a), which means that when $\mu<\mu_{c}\left(\mu>\mu_{c}\right)$ increasing $\alpha$ value could enhance (shrink) the nonlinearity and concentrate (disperse) the energy of the considered plasma, so the pulses become taller (shorter). For $s=-1$ and $q>0$, the amplitude of ion-acoustic rogue wave shrinks with an increase in $\alpha$, which is presented in Plot (b). We speculate that for this case, increasing $\alpha$ reduces the nonlinearity of the system and disperses the energy of the rogue waves, which makes the pulses become shorter.

(iv) From plots (c) and (d) in Fig. 9, it is revealed the effect of $\alpha$ on the amplitude for $s=+1$, i.e. the system containing positive dust, is opposite to that for $s=-1$, i.e. the system containing negative dust.

We can also analyze the effects of other plasma parameters on the nonlinear structures of ionacoustic rogue wave (28) by the similar procedures, and therefore we omit them here for simplicity.

\subsection{MI of cylindrical and spherical excitations}

When setting $v=1$ and 2 in Eqs. (20), (25) and (26), we can get the modified NLSE, nonlinear dispersion relation and MI growth rate for cylindrical and spherical excitations, respectively. 
a

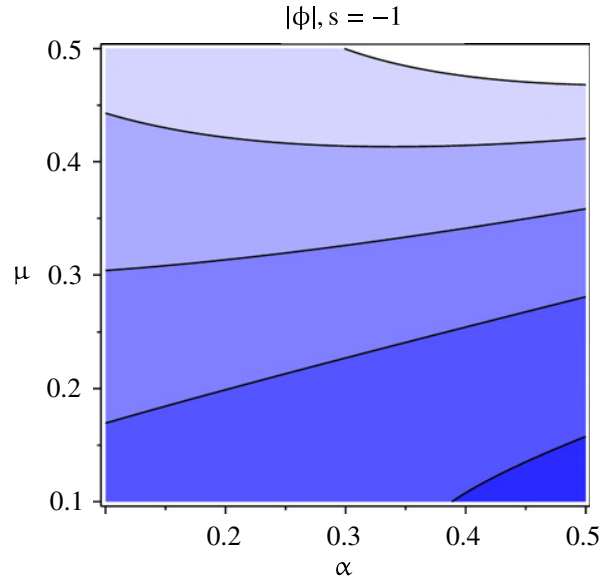

c

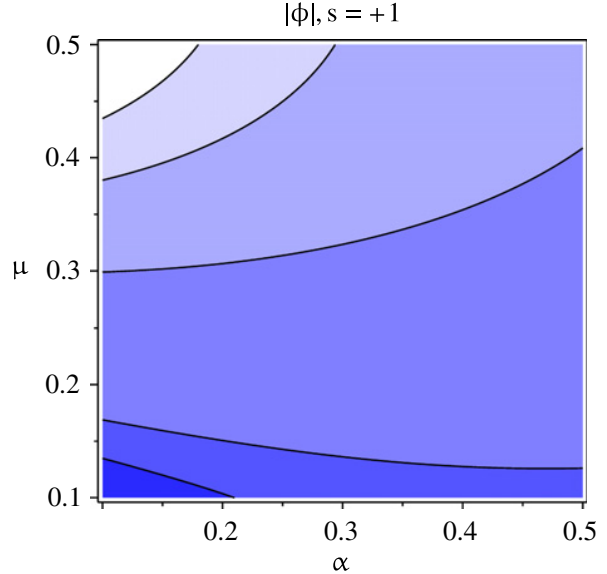

$\mathrm{b}$

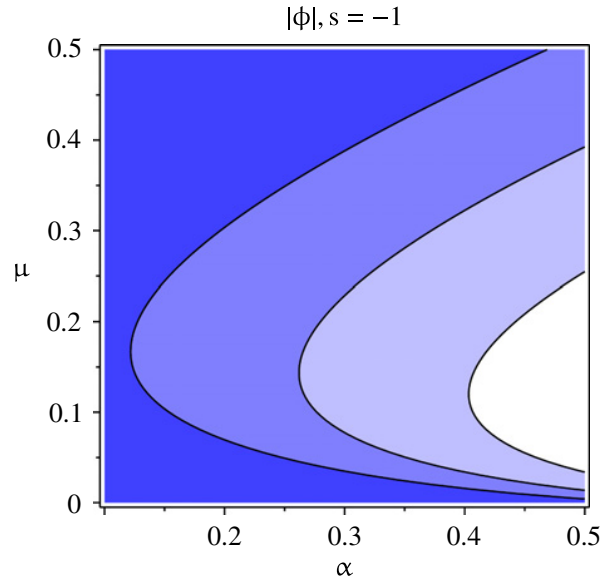

d

$|\phi|, \mathrm{s}=+1$

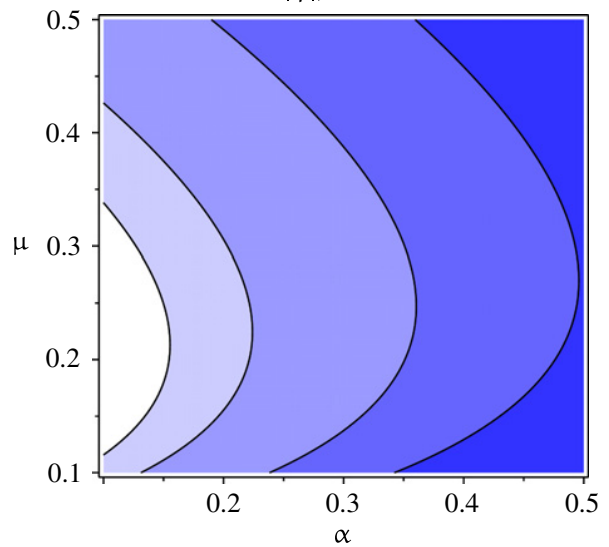

Fig. 9. The contour of the second-order ion-acoustic rogue wave amplitude. The lighter-color regions correspond to the higher values of amplitude. The parameters are: $\beta=0.2, \gamma=0.4, \delta=0.1, k=2.5$, (a), $q=-0.9, s=-1$, (b), $q=1.5, s=-1$, (c), $q=-0.9, s=+1,(\mathrm{~d}), q=1.5, s=+1$.

From Eq. (26), it is clear that the MI growth will cease for $v=1$ (cylindrical geometry) when

$$
\tau \geq \tau_{\max }=\frac{2 b}{a X^{2}}
$$

and for $v=2$ (spherical geometry) when

$$
\tau \geq \tau_{\max }=\frac{1}{X} \sqrt{\frac{2 b}{a}} .
$$

We can see that there is a MI period for nonplanar (cylindrical and spherical geometries) wave modulation, which does not exist in the planar case.

In Fig. 10, we present how the parameter $q$ affects the MI growth rate $\Gamma$ and the maximum period $\tau_{\max }$ for the cylindrical excitation. From plots (a) and (b), we can see that: (i) for $s=-1$, both $\Gamma$ and $\tau_{\max }$ increase as the values of $q \in\left(-1, q_{c}\right)$ increase; then, beyond a certain value $q_{c}$, increasing $q$ value leads to the decrease of both $\Gamma$ and $\tau_{\max }$. If the system contains positive dust $(s=+1)$, the parameter 
$\mathrm{a}$

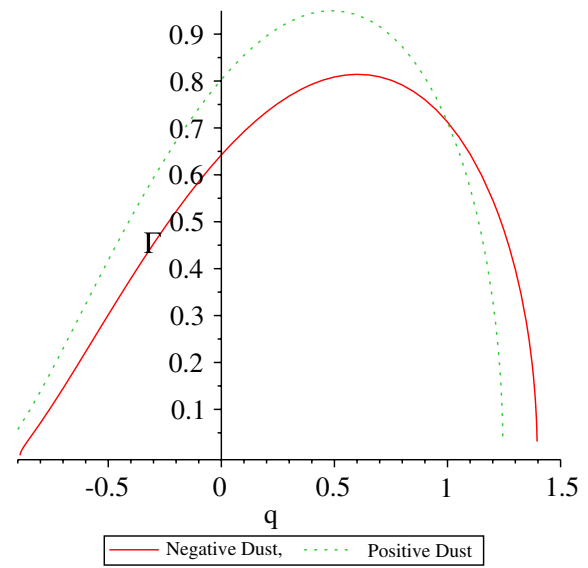

b

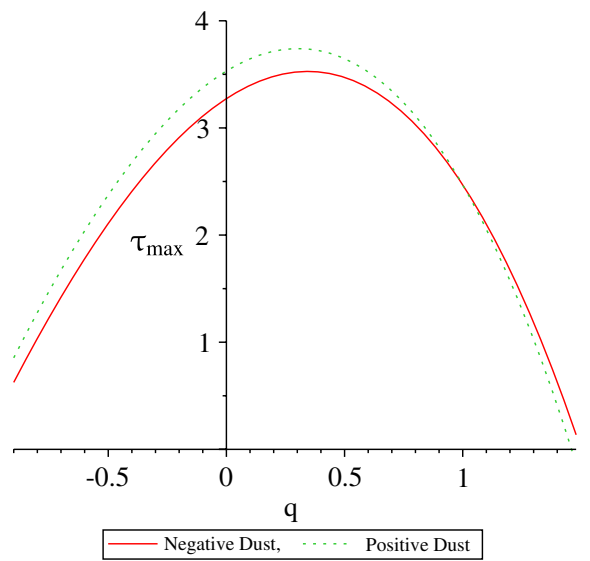

Fig. 10. MI growth rate $\Gamma$ (a) and the maximum period $\tau_{\max }$ (b) for cylindrical excitation. The parameters are: (a), $\alpha=0.4, \beta=$ $0.07, \gamma=0.1, \mu=0.6, \delta=0.1, k=1.5, v=1, X=0.4$, and $\tau=0.6$; (b) $\alpha=0.2, \beta=0.03, \gamma=0.4, \mu=0.5, \delta=$ $0.2, k=1.7, v=1$, and $X=0.9$.

$q$ has the same qualitative influence on the MI growth rate $\Gamma$ and the maximum period $\tau_{\max }$. (ii) when $q<q_{c^{\prime}}=1$, the values of $\Gamma$ and $\tau_{\max }$ for $s=-1$ are smaller than that for $s=+1$; however, both the $\Gamma$ and $\tau_{\max }$ values for $s=-1$ are bigger than that for $s=+1$ if $q>q_{c^{\prime}}$. For the spherical excitation, the nonextensive index $q$ has the same qualitative behavior on the MI growth rate $\Gamma$ and the maximum period $\tau_{\max }$ as Fig. 10, so we do not include these figures here.

The growth $\Lambda$ of the modulation during the unstable period is defined as

$$
\Lambda=\exp \left(\int_{\tau_{0}}^{\tau_{\max }} \Gamma(\tau) d \tau\right)=\exp \left(\frac{Q}{\tau_{0}^{\nu-1}} f(R)\right)
$$

where $R=\frac{2 b}{a X^{2} \tau_{0}^{v}}>1$. From Eqs. (29) and (30), we can see that the condition of the modulational instability growth will be satisfied if $R>1$. Here,

$$
f(R)= \begin{cases}f_{1}(R)=\arctan \sqrt{R-1}-\frac{\sqrt{R-1}}{R}, & v=1, \\ f_{2}(R)=\frac{1}{R}\left(\sqrt{R} \ln \frac{\sqrt{R}+\sqrt{R-1}}{\sqrt{R}-\sqrt{R-1}-2 \sqrt{R-1}),}, v=2 .\right.\end{cases}
$$

Form the above equation, we can see that $f_{1}(R)$ is an increasing function of $R$, and $f_{1}(R) \rightarrow \frac{\pi}{2}$ as $R \rightarrow+\infty$, which means that the total modulation growth $\Lambda$ increases as $R$ does for cylindrical excitation. But $f_{2}(R)$ has a maximum value $f_{2}\left(R_{c}\right)=\frac{2 \sqrt{R_{c}-1}}{R_{c}}$. Here, $R_{c}$ is determined by $4 \sqrt{R_{c}-1}=$ $\sqrt{R_{c}} \ln \frac{\sqrt{R_{c}}+\sqrt{R_{c}-1}}{\sqrt{R_{c}}-\sqrt{R_{c}-1}}$. In other words, for $1<R<R_{c}$, the total modulation growth $\Lambda$ is an increasing function in $R$ and then decreases as $R$ increases further.

Fig. 11 shows how the parameter $q$ affects the growth $\Lambda$. From this figure, we can see that: (i) for the case $s=-1, v=1$, the growth $\Lambda$ is a decreasing (an increasing) function in $q$ if $-1<q<q_{c}\left(q>q_{c}\right)$. For other cases, i.e. $(s=-1, v=2)$, and $(s=+1, v=1,2)$, the parameter $q$ has the similar qualitative influence on the growth $\Lambda$. (ii) when $s=-1$, the growth $\Lambda$ value for $v=1$ is smaller than that for $v=2$ except that $q \rightarrow-1$. It can be seen that this interesting phenomenon also appears in the case $s=+1$. (iii) when $v=1$, the value of growth $\Lambda$ for $s=-1$ is bigger than that for $s=+1$ except that $q$ reaches the critical value. Also the same behavior appears in the case $v=2$. 


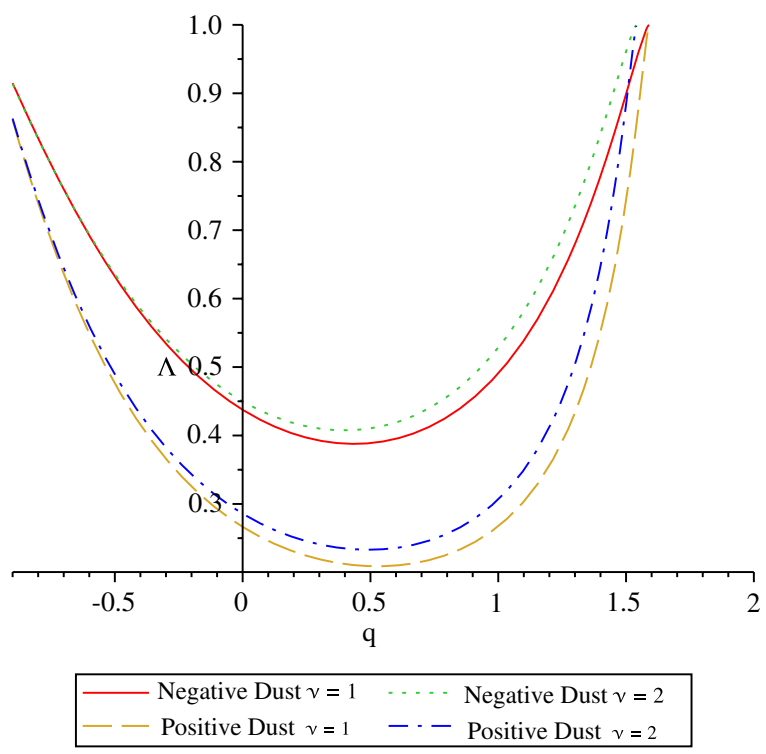

Fig. 11. The growth $\Lambda$ of the modulation. The parameters are: (a), $\alpha=0.35, \beta=0.04, \gamma=0.2, \mu=0.7, \delta=0.2, k=$ $2, X=0.6$, and $\tau_{0}=0.25$.

\section{Conclusions}

In summary, we investigated the modulational instability of the planar and nonplanar (cylindrical and spherical) ion-acoustic waves in a multicomponent plasma consisting of positive ions, nonextensive positrons and electrons with two temperature distributions, as well as charged dust grains. Both negative and positive dust charge cases were considered. Via the RPM, a modified nonlinear Schrödinger equation was derived for the wave amplitude.

For the planar excitations, the effects of the plasma parameters, especially the nonextensive index $q$, dust concentration $\alpha$ and cold electron to positive ion density ratio $\mu$ on the critical wave number threshold $k_{c}$ which determines where the stable $(P Q<0)$ and unstable $(P Q>0)$ regions set in, were discussed in detail. The effect of the $q$ on the MI growth rate was also studied. Within the modulational unstable envelope pulse region, the external perturbations may lead to the creation of ion-acoustic rogue waves. Our results revealed that the parameter $q$ plays a significant roles in changing the energy of rogue waves. For different values of $q$, other plasma parameters, $\mu$ and $\alpha$, for example, have different influences on the nonlinear structures of rogue waves. As far as we know, the second-order ionacoustic rogue waves were studied for the first time. In the real applications, we can control (and in fact prevent) the energetic pulses of the rogue waves by changing the values of these parameters.

For the cylindrical and spherical wave modulation, we found that there is a modulation instability period which does not exist in the planar geometry. We also analyzed how the nonextensive parameter $q$ affects the modulational instability growth rate $\Gamma$, the maximum growth period $\tau_{\max }$ and the total growth $\Lambda$.

Our investigation may provide a better understanding of the nonlinear wave phenomena in laboratory experiments and in interstellar and spatial observations, where nonextensive plasma distributions may be present.

\section{Acknowledgments}

The authors express their sincere thanks to the referees for their valuable suggestions which led to an improved version. 


\section{Appendix. Expressions of the coefficients}

$$
\begin{aligned}
\Delta_{1}= & \frac{k^{2}\left(2 \delta c_{2} \gamma^{2} \omega^{2}-2 \mu c_{2} U^{2} \omega^{2}-2 \eta c_{2} W^{2} \omega^{2}+3 k^{2} \mu c_{1} U+3 k^{2} \eta c_{1} W+3 k^{2} \delta c_{1} \gamma+12 k^{4}\right)}{2 \omega^{2}\left(-k^{2}+\omega^{2} \mu c_{1} U+\omega^{2} \eta c_{1} W+\omega^{2} \delta c_{1} \gamma+4 k^{2} \omega^{2}\right)}, \\
\Delta_{2}= & \frac{1}{2 \omega^{3}\left(-k^{2}+\omega^{2} \mu c_{1} U+\omega^{2} \eta c_{1} W+\omega^{2} \delta c_{1} \gamma+4 k^{2} \omega^{2}\right)} \\
& \times\left[k \left(2 k^{4}+2 \delta c_{2} \gamma^{2} \omega^{4}-2 \mu c_{2} U^{2} \omega^{4}-2 \eta c_{2} W^{2} \omega^{4}\right.\right. \\
& \left.\left.+k^{2} \omega^{2} \mu c_{1} U+k^{2} \omega^{2} \eta c_{1} W+k^{2} \omega^{2} \delta c_{1} \gamma+4 k^{4} \omega^{2}\right)\right], \\
\Delta_{3}= & \frac{3 k^{4}+2 \delta c_{2} \gamma^{2} \omega^{4}-2 \mu c_{2} U^{2} \omega^{4}-2 \eta c_{2} W^{2} \omega^{4}}{2 \omega^{2}\left(-k^{2}+\omega^{2} \mu c_{1} U+\omega^{2} \eta c_{1} W+\omega^{2} \delta c_{1} \gamma+4 k^{2} \omega^{2}\right)} \\
\Delta_{4}= & \frac{1}{\omega^{3}\left(c_{1} V^{2} \mu U+c_{1} V^{2} \eta W+c_{1} V^{2} \delta \gamma-1\right)} \\
& \times\left[2 c_{1} V k^{3} \mu U+2 c_{1} V k^{3} \eta W+2 c_{1} V k^{3} \delta \gamma-2 \eta c_{2} W^{2} \omega^{3}\right. \\
& \left.+2 \delta c_{2} \gamma^{2} \omega^{3}-2 \mu c_{2} U^{2} \omega^{3}+\omega c_{1} k^{2} \mu U+\omega c_{1} k^{2} \eta W+\omega c_{1} k^{2} \delta \gamma\right] \\
\Delta_{5}= & \frac{1}{\omega^{3}\left(c_{1} V^{2} \mu U+c_{1} V^{2} \eta W+c_{1} V^{2} \delta \gamma-1\right)} \\
& \times\left[-2 V \eta c_{2} W^{2} \omega^{3}+2 V \delta c_{2} \gamma^{2} \omega^{3}-2 V \mu c_{2} U^{2} \omega^{3}+V \omega c_{1} k^{2} \mu U\right. \\
& \left.+V \omega c_{1} k^{2} \eta W+V \omega c_{1} k^{2} \delta \gamma+2 k^{3}\right] \\
\Delta_{6}= & \frac{2 V^{2} \eta c_{2} W^{2} \omega^{3}+2 V^{2} \delta c_{2} \gamma^{2} \omega^{3}-2 V^{2} \mu c_{2} U^{2} \omega^{3}+2 V k^{3}+k^{2} \omega}{\omega^{3}\left(c_{1} V^{2} \mu U+c_{1} V^{2} \eta W+c_{1} V^{2} \delta \gamma-1\right)} .
\end{aligned}
$$

Here,

$$
\begin{aligned}
& V=(\delta \gamma+U \mu+W \eta) c_{1} \frac{\omega^{3}}{k^{3}}, \quad c_{1}=\frac{(q+1)}{2}, \quad c_{2}=\frac{(q+1)(3-q)}{8}, \\
& U=\frac{1}{\mu+\eta \beta}, \quad W=\frac{\beta}{\mu+\eta \beta} .
\end{aligned}
$$

\section{References}

[1] H.R. Miller, P.J. Witta (Eds.), Active Galactic Nuclei, Springer, Berlin, 1998.

[2] F.C. Michel, Theory of Neutron Star Magnetospheres, Chicago University Press, Chicago, 1991.

[3] R. Schlickeiser, P.K. Shukla, Astrophys. J. 599 (2003) L57.

[4] M.J. Rees, Nature 229 (1971) 312.

[5] R.G. Greaves, C.M. Surko, Phys. Rev. Lett. 75 (1995) 3846.

[6] Laser creates billions of antimatter particles. Cosmos Online: http://www.cosmosmagazine.com/news/2345/ laser-creates-billions-particles-antimatter?page $=0 \% 2 \mathrm{CO}$.

[7] A.A. Mamun, P.K. Shukla, Geophys. Res. Lett. 29 (2002) 1870.

[8] M. Tribeche, A. Merriche, Phys. Plasmas 18 (2011) 033706.

[9] W.M. Moslem, Phys. Lett. A 351 (2006) 290.

[10] B. Tian, Y.T. Gao, Phys. Plasmas 12 (2005) 054701.

[11] A. Barkan, N. D’Angelo, R. Merlino, Planet. Space Sci. 44 (1996) 239.

[12] S.A. El-Tantawy, N.A. El-Bedwehy, W.M. Moslem, Phys. Plasmas 18 (2011) 052113.

[13] S. Ghosh, R. Bharuthram, Astrophys. Space Sci. 314 (2008) 121.

[14] W.M. Moslem, R. Sabry, S.K. El-Labany, P.K. Shukla, Phys. Rev. E 84 (2011) 066402.

[15] J.R. Asbridge, S.J. Bame, I.B. Strong, J. Geophys. Res. 73 (1968) 5777.

[16] R. Lundlin, et al., Nature 341 (1989) 609.

[17] Y. Futaana, et al., J. Geophys. Res. 108 (2003) 151.

[18] J.M. Liu, et al., Phys. Rev. Lett. 72 (1994) 2717.

[19] A. Renyi, Acta Math. Acad. Sci. Hungar. 6 (1955) 285.

[20] C. Tsallis, J. Stat. Phys. 52 (1988) 479.

[21] R.F. Aranha, I.D. Soares, E.V. Tonini, Phys. Rev. D 81 (2010) 104005.

[22] H.P. de Oliveira, I.D. Soares, E.V. Tonini, Phys. Rev. D 67 (2003) 063506.

[23] S. Guo, H. Wang, L. Mei, Phys. Plasmas 19 (2012) 063701.

[24] J.A.S. Lima, R. Silva Jr., J. Santos, Phys. Rev. E 61 (2000) 3260.

[25] B. Liu, J. Goree, Phys. Rev. Lett. 100 (2008) 055003.

[26] M.P. Leubner, Astrophys. Space Sci. 282 (2002) 573.

[27] D. Summers, R.M. Thorne, Phys. Fluids B 3 (1991) 1835. 
[28] M. Temerin, K. Cerny, W. Lotko, F.S. Mozer, Phys. Rev. Lett. 48 (1982) 1175.

[29] Y. Nishida, T. Nagasawa, Phys. Fluids 29 (1986) 345.

[30] N.L. Shatashvili, J.I. Javakhishvili, H. Kaya, Astrophys. Space Sci. 250 (1997) 109.

[31] R. Sabry, W.M. Moslem, P.K. Shukla, H. Saleem, Phys. Rev. E 79 (2009) 056402.

[32] M.K. Mishra, R.S. Tiwari, S.K. Jain, Phys. Rev. E 76 (2007) 036401.

[33] T. Taniuti, N. Yajima, J. Math. Phys. 10 (1969) 1369.

[34] C. Kharif, E. Pelinovsky, A. Slunyaev, Rogue Waves in the Ocean, Springer-Verlag, Berlin, 2009.

[35] P.K. Shukla, I. Kourakis, B. Eliasson, M. Marklund, L. Stenflo, Phys. Rev. Lett. 97 (2006) 094501.

[36] N. Akhmediev, A. Ankiewicz, Solitons, Nonlinear Pulses and Beams, Chapman and Hall, London, 1997.

[37] W.M. Moslem, R. Sabry, S.K. El-Labany, P.K. Shukla, Phys. Rev. E 84 (2011) 066402.

[38] A. Chabchoub, N.P. Hoffmann, N. Akhmediev, Phys. Rev. Lett. 106 (2011) 204502.

[39] A. Chabchoub, N. Akhmediev, N.P. Hoffmann, Phys. Rev. E 86 (2012) 016311.

[40] A. Chabchoub, N. Hoffmann, M. Onorato, N. Akhmediev, Phys. Rev. X 2 (2012) 011015.

[41] D.H. Peregrine, J. Aust. Math. Soc. B 25 (1983) 16.

[42] N. Akhmediev, V.I. Korneev, Theoret. Math. Phys. 69 (1986) 1089.

[43] N. Akhmediev, A. Ankiewicz, M. Taki, Phys. Lett. A 373 (2009) 675.

[44] D.R. Solli, C. Ropers, P. Koonath, B. Jalali, Nature (London) 450 (2007) 1054.

[45] D-Il. Yeom, B. Eggleton, Nature (London) 450 (2007) 953.

[46] B. Kibler, et al., Nat. Phys. 6 (2010) 790.

[47] H. Bailung, S.K. Sharma, Y. Nakamura, Phys. Rev. Lett. 107 (2011) 255005.

[48] R. Silva Jr., A.R. Plastino, J.A.S. Lima, Phys. Lett. A 249 (1998) 401.

[49] R. Sabry, S.K. El-Labany, P.K. Shukla, Phys. Plasmas 15 (2008) 122310.

[50] N.S. Saini, I. Kourakis, Phys. Plasmas 15 (2008) 123701.

[51] Y.C. Ma, Stud. Appl. Math. 60 (1979) 43.

[52] E. Kuznetsov, Dokl. Akad. Nauk SSSR 235 (1977) 575.

[53] N. Akhmediev, V.I. Korneev, Theoret. Math. Phys. 69 (1986) 1089.

[54] Z. Yan, Phys. Lett. A 374 (2010) 672. 